\title{
Produtividade de cana soca sem queima em função do uso de gesso e vinhaça ${ }^{1}$
}

\author{
Juliana Mariano Carvalho ${ }^{2}$, Marcelo Andreotti ${ }^{2}$, Salatiér Buzetti ${ }^{2}$, Morel de Passos e Carvalho ${ }^{2}$
}

\begin{abstract}
Unburned ratoon sugarcane yield according to gypsum and vinasse doses

Understanding the influence of sugarcane straw over the application dynamic of vinasse and correctives is essential for an effective management of the production system. This study aimed at evaluating soil fertility, stalk yield and technological quality of ratoon sugarcane (2nd cycle), according to vinasse and gypsum application, in an Oxisol, in the Northwest São Paulo State, Brazil. The study was carried out with the RB867515 cultivar and the experimental design was randomized blocks with four replications, in a $2 \times 3$ factorial scheme, i.e., with and without gypsum $\left(1.0 \mathrm{t} \mathrm{ha}^{-1}\right)$ and vinasse application in three doses: $0.0 \mathrm{~m}^{3} \mathrm{ha}^{-1}$ (control), $100.0 \mathrm{~m}^{3} \mathrm{ha}^{-1}$ and $200.0 \mathrm{~m}^{3} \mathrm{ha}^{-1}$. The gypsum application increased the $\mathrm{Ca}^{2+}, \mathrm{Mg}^{2+}, \mathrm{SO}_{4}^{2-}, \mathrm{pH}$ and base saturation and reduced the contents of $\mathrm{Al}^{3+}$, in the $0-0.20 \mathrm{~m}$ and $0.20-0.40 \mathrm{~m}$ layers, and increased the $\mathrm{SO}_{4}{ }^{2-}$ content in the 0.40-0.60 m layer, after 180 and 360 days, respectively. The gypsum plastering and the vinasse application did not increase the stalk yield nor improved its technologic quality.
\end{abstract}

KEY-WORDS: Saccharum spp.; gypsum plastering; aluminium; plant biomass.

\section{INTRODUÇÃO}

A cana-de-açúcar, por tratar-se de uma cultura semiperene, pode ficar no campo por vários anos (média de quatro a sete anos, em áreas de produção de usinas), sem, muitas vezes, a devida correção de acidez do solo e/ou manutenção de adubação adequada, a cada safra, que, normalmente, são realizadas de modo mais criterioso, quando ocorre reforma do canavial. Este fato não anula a necessidade de manutenção de sua fertilidade, pois, com o passar dos anos, os valores de $\mathrm{pH}$ vão diminuindo, levando à redução da saturação por bases do solo e aumento dos teores de Al trocável (Rossetto et al. 2008).

\section{RESUMO}

A compreensão da influência da palhada de cana-deaçúcar sobre a dinâmica da aplicação de vinhaça e corretivos é essencial para o bom gerenciamento do sistema produtivo. O objetivo da realização deste trabalho foi avaliar os teores de nutrientes do solo, produtividade de colmos e a qualidade tecnológica da cana soca ( $2^{\circ}$ ciclo), em função da aplicação de vinhaça e gesso, em Latossolo Vermelho-Amarelo distrófico do noroeste paulista. $\mathrm{O}$ trabalho foi desenvolvido com a cultivar RB867515 e o delineamento experimental foi em blocos casualizados, com quatro repetições, em esquema fatorial $2 \times 3$, ou seja, com e sem gessagem $\left(1,0 \mathrm{t} \mathrm{ha}^{-1}\right)$ e aplicação de vinhaça em três doses: $0,0 \mathrm{~m}^{3} \mathrm{ha}^{-1}$ (testemunha), 100,0 $\mathrm{m}^{3} \mathrm{ha}^{-1}$ e $200,0 \mathrm{~m}^{3} \mathrm{ha}^{-1}$. O uso de gesso incrementou os teores de $\mathrm{Ca}^{2+}$, $\mathrm{Mg}^{2+} \mathrm{e} \mathrm{SO}_{4}{ }^{2-}$ e valores de $\mathrm{pH} \mathrm{e} \mathrm{V \%}$, bem como reduziu os teores de $\mathrm{Al}^{3+}$, nas camadas de $0-0,20 \mathrm{~m}$ e $0,20-0,40 \mathrm{~m}$, e aumentou o teor de $\mathrm{SO}_{4}{ }^{2-}$, na camada de 0,40-0,60 m, aos 180 e 360 dias, respectivamente. A gessagem e a aplicação de vinhaça não resultaram em aumento na produtividade de colmos, ou melhoria da qualidade tecnológica.

PALAVRAS-CHAVES: Saccharum spp.; gessagem; alumínio; biomassa vegetal.

No sistema de colheita mecanizada, grande quantidade de palha é depositada no solo, tornando-se reserva de nutrientes, devido à decomposição da sua matéria orgânica (Oliveira et al. 1999). Luca et al. (2008) observaram taxa média de 13,3 tha ${ }^{-1}$ ano $^{-1}$ de entrada de palha no sistema de colheita da cana sem queima. A presença desta palhada pode alterar a dinâmica nutricional da planta e requer estudos, quanto à aplicação de fertilizantes orgânicos ou minerais, corretivos e outros insumos.

Os solos da região noroeste paulista apresentam $\mathrm{pH}$ baixo e podem proporcionar toxidez por $\mathrm{Al}^{3+}$ e/ou deficiência de cálcio, restringindo o desenvolvimento das raízes em profundidade. Por

1. Trabalho recebido em maio/2012 e aceito para publicação em fev./2013 ( ${ }^{\circ}$ registro: PAT 18633).

2. Universidade Estadual Paulista (Unesp), Faculdade de Engenharia, Departamento de Fitossanidade, Engenharia Rural e Solos, Ilha Solteira, SP, Brasil. E-mails: julianamaricar@hotmail.com, dreotti@agr.feis.unesp.br, sbuzetti@agr.feis.unesp.br, morel@agr.feis.unesp.br. 
isto, o uso do gesso agrícola apresenta-se como uma boa alternativa para melhorar a estrutura e a disponibilidade de nutrientes no solo, nas camadas mais profundas, favorecendo o aumento da produtividade e a longevidade do canavial (Raij 2008a).

Rocha (2007) avaliou a resposta do gesso mineral na correção do alumínio trocável e na ocorrência de lixiviação de bases, em um latossolo, e concluiu que a aplicação do gesso foi eficaz no fornecimento de cálcio em subsuperfície e que a utilização de doses de até $3,42 \mathrm{tha}^{-1}$ reduziu em $39 \%$ a saturação por alumínio do solo. Desta forma, o gesso agrícola facilita a remoção de bases como o magnésio e o potássio para a subsuperfície do solo, bem como fornece cálcio e enxofre e diminui a toxidez por alumínio, nas camadas subsuperficiais (Saldanha et al. 2007, Raij 2008b), induzindo a uma maior profundidade do sistema radicular e longevidade da cultura da cana-de-açúcar.

Outra prática comum em sistemas de produção da cana-de-açúcar é a aplicação de vinhaça ao solo. A vinhaça é o principal subproduto da produção de etanol e é fonte de matéria orgânica e nutrientes, principalmente potássio (Resende et al. 2006). Canella et al. (2003) constataram que a vinhaça alterou as propriedades químicas do solo e proporcionou melhoria à fertilidade e à quantidade da matéria orgânica do solo. Brito et al. (2009), estudando o comportamento da vinhaça em diferentes solos, verificaram que o aumento do teor de K no solo variou em profundidade, de acordo com as particularidades de cada solo. Sendo assim, a aplicação deste subproduto em solos agrícolas pode alterar a dinâmica do ciclo dos elementos no solo, modificando a fertilidade e a nutrição das plantas (Chiaradia 2005).

Considerando-se o efeito da palha remanescente de cortes anteriores, principalmente em áreas na região noroeste do Estado de São Paulo, objetivou-se avaliar o efeito da aplicação de vinhaça e gesso nos teores de nutrientes no solo, em um Latossolo Vermelho-Amarelo, e a produtividade de cana soca $\left(2^{\circ}\right.$ ciclo $)$.

\section{MATERIAL E MÉTODOS}

O experimento foi desenvolvido na safra 2009/2010, em área administrada pela Usina Vale do Paraná, localizada em Suzanápolis (SP). As coordenadas geográficas na área, segundo dados fornecidos pela Usina, são $20^{\circ} 32^{\prime} 02^{\prime}$ 'S e $50^{\circ} 58^{\prime} 28^{\prime \prime} \mathrm{W}$, com altitude de 360,0 m.
A pluviosidade e a temperatura média anual da região são, respectivamente, $1.217,0 \mathrm{~mm}$ e $23,7^{\circ} \mathrm{C}$, sendo o tipo climático classificado como $\mathrm{Aw}$, segundo caracterização internacional de Köppen. Os dados de precipitação pluvial e temperatura, durante o período de condução do experimento, são apresentados na Figura 1.

$\mathrm{O}$ experimento foi instalado em uma área de primeira cana soca $\left(2^{\circ}\right.$ ciclo), cultivar RB867515. A escolha desta variedade deveu-se à sua adaptação e grande cultivo na região noroeste de São Paulo (Udop 2007).

Antes do plantio da cana (março de 2008), a área encontrava-se com pastagem de braquiária, em cultivo extensivo há mais de 20 anos, a qual foi dessecada com herbicida glyphosate, na dose de $2,12 \mathrm{~kg}$ do i.a. ha ${ }^{-1}$.

Ainda antes do plantio da cana, conforme padronização da Usina, o preparo do solo foi realizado com uma gradagem pesada e duas gradagens intermediárias, para destruição dos restos vegetais da pastagem. Nesta gradeação intermediária, foi aplicada 1,0 t de calcário (PRNT de 85\%), com incorporação com arado de aiveca, a $0,35 \mathrm{~m}$ de profundidade, sendo finalizada com uma grade niveladora. Na sulcação, adubou-se a área com $500,0 \mathrm{~kg} \mathrm{ha}^{-1}$ da fórmula 06-30-24, e, na cobertura dos toletes, foram aplicados, no sulco de plantio, 250,0 g do i.a. ha $^{-1}$ de fipronil, visando ao controle de pragas do solo.

O solo da área experimental foi caracterizado como Latossolo Vermelho-Amarelo distrófico, textura média (Embrapa 2006). Cada unidade experimental (parcela) foi constituída por quatro linhas de cana-de-açúcar, em 15,0 m de comprimento, espaçadas

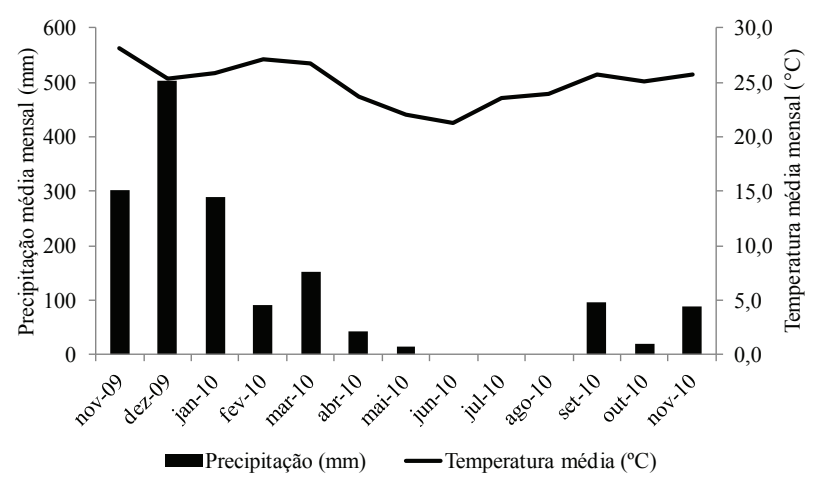

Figura 1. Precipitação pluvial e temperatura, de novembro de 2009 a novembro de 2010, na área experimental (Suzanápolis, SP, 2010). 
em 1,50 m entre as linhas, perfazendo $90,0 \mathrm{~m}^{2}$. Para a avaliação dos dados, como parcela útil, foram utilizadas as duas linhas centrais, desprezando-se 1,0 m em cada extremidade.

Inicialmente, no dia 19/09/2009, antes da aplicação dos tratamentos, foi realizada a caracterização química (Raij \& Quaggio 1983) e granulométrica (Embrapa 1997) do solo, nas profundidades de $0-0,20 \mathrm{~m}, 0,20-0,40 \mathrm{~m}$ e $0,40-0,60 \mathrm{~m}$ (Tabela 1 ), coletando-se 20 amostras simples, com um trado de rosca, nas entrelinhas das parcelas.

O delineamento experimental utilizado foi em blocos casualizados, com quatro repetições, em esquema fatorial $2 \times 3$. Os tratamentos foram constituídos de parcelas com e sem a aplicação de gesso e da aplicação de três doses de vinhaça: $0,0 \mathrm{~m}^{3} \mathrm{ha}^{-1}$ (testemunha), 100,0 $\mathrm{m}^{3} \mathrm{ha}^{-1}$ e 200,0 $\mathrm{m}^{3} \mathrm{ha}^{-1}$.

O gesso foi aplicado a lanço, sem incorporação, no dia 01/10/2009 (90 dias após a colheita da cana planta), sendo a dose de gesso de 1,0 $\mathrm{t} \mathrm{ha}^{-1}$ calculada pelo teor de argila do solo, na camada de 0,20-0,40 m, conforme recomendação de Raij \& Cantarella (1996), e aproximada para a operacionalidade da Usina. Nesta mesma data, foi realizada adubação de soqueira, com 400,0 $\mathrm{kg} \mathrm{ha}^{-1}$ do formulado 20-0-30.

As doses de vinhaça, fornecidas pela própria Usina Vale do Paraná, foram aplicadas aos 41 dias após a aplicação do gesso (11/11/2009), utilizando-se um aplicador motorizado, com controle de vazão pressurizado, com correção da quantidade a ser aplicada pela vazão e área total de cada parcela. A determinação da composição química da vinhaça foi realizada pelo laboratório de química da Escola Superior de Agricultura Luiz de Queiroz da Universidade de São Paulo (Tabela 2).

As avaliações para a caracterização química do solo foram realizadas aos 180 e 360 dias após a aplicação dos tratamentos (DAAT), nas camadas de 0-0,20 m, 0,20-0,40 m e 0,40-0,60 m, segundo método descrito por Raij \& Quaggio (1983). Para tanto, foram coletadas dez amostras simples de solo nas entrelinhas da cana soca, por camada e por parcela, utilizando-se um trado de rosca.

Para a avaliação do teor nutricional das folhas, foram coletadas as folhas +3 , correspondentes à primeira folha a partir do ápice com o "colarinho" totalmente visível, sendo retiradas 20 folhas por parcela, coletadas aos 100 DAAT (22/02/2009). As folhas foram cortadas, descartando-se as pontas e a base e tomando-se os 0,40 $\mathrm{m}$ da parte central, com exclusão da nervura, e colocadas para secar em estufa com circulação forçada de ar $\left(65^{\circ} \mathrm{C}\right)$, por 72 horas, em média. Depois de seco, o material foi moído e foram determinados os teores de $\mathrm{N}, \mathrm{P}, \mathrm{K}, \mathrm{Ca}, \mathrm{Mg}$ e $\mathrm{S}$, seguindo-se os métodos descritos por Malavolta et al. (1997).

A determinação do número de colmos foi realizada aos 408 DAAT (23/11/2010), sendo os colmos contados em 2,0 m lineares, nas duas linhas centrais da parcela. Após a contagem, estes foram cortados a

Tabela 1. Atributos químicos e granulometria do solo, antes da aplicação de gesso e vinhaça, nas diferentes camadas (Suzanápolis, SP, 2010).

\begin{tabular}{|c|c|c|c|c|c|c|c|c|c|c|c|c|c|c|c|c|}
\hline Profundidade & $\mathrm{P}$ & M.O. & \multirow{2}{*}{$\begin{array}{c}\mathrm{pH} \\
\left(\mathrm{CaCl}_{2}\right)\end{array}$} & $\mathrm{K}$ & $\mathrm{Ca}$ & $\mathrm{Mg}$ & $\mathrm{H}+\mathrm{Al}$ & $\mathrm{Al}$ & $\mathrm{S}-\mathrm{SO}_{4}$ & SB & CTC & V & $\mathrm{m}$ & Argila & Silte & Areia \\
\hline $\mathrm{m}$ & $\mathrm{mg} \mathrm{dm}{ }^{-3}$ & $\mathrm{~g} \mathrm{dm}^{-3}$ & & \multicolumn{8}{|c|}{$\mathrm{cmol}_{\mathrm{c}} \mathrm{dm}^{-3}$} & \multicolumn{2}{|c|}{$-\%$} & \multicolumn{3}{|c|}{$\mathrm{g} \mathrm{kg}^{-1}$} \\
\hline $0,00-0,20$ & 3 & 11 & 4,4 & 0,2 & 5 & 3 & 26 & 5 & 1 & 8,2 & 34,2 & 24 & 38 & 125 & 53 & 822 \\
\hline $0,20-0,40$ & 3 & 10 & 4,2 & 0,1 & 4 & 2 & 28 & 8 & 1 & 6,1 & 34,1 & 18 & 57 & 139 & 58 & 802 \\
\hline $0,40-0,60$ & 3 & 11 & 4,2 & 0,1 & 4 & 2 & 31 & 10 & 2 & 6,1 & 37,1 & 16 & 62 & 165 & 60 & 774 \\
\hline
\end{tabular}

Tabela 2. Composição química da vinhaça (Suzanápolis, SP, 2009).

\begin{tabular}{|c|c|c|c|c|c|c|c|c|c|c|c|c|c|c|}
\hline \multirow{2}{*}{$\begin{array}{c}\mathrm{pH} \\
\mathrm{H}_{2} \mathrm{O}\end{array}$} & Densidade & \multirow{2}{*}{$\begin{array}{l}\text { Resíduo } \\
\text { Seco } 100- \\
110^{\circ} \mathrm{C}\end{array}$} & M.O. & $\mathrm{N}$ & $\mathrm{P}_{2} \mathrm{O}_{5}$ & $\mathrm{~K}_{2} \mathrm{O}$ & $\mathrm{Ca}$ & $\mathrm{Mg}$ & $\mathrm{S}$ & $\mathrm{Cu}$ & $\mathrm{Mn}$ & $\mathrm{Zn}$ & $\mathrm{Fe}$ & \multirow[t]{2}{*}{$\mathrm{C} / \mathrm{N}$} \\
\hline & $\mathrm{kg} \mathrm{dm}^{-3}$ & & \multicolumn{7}{|c|}{$\mathrm{g} \mathrm{dm}^{-3}$} & \multicolumn{4}{|c|}{$\mathrm{mg} \mathrm{dm}^{-3}$} & \\
\hline 3,9 & 1,0 & 16,9 & 12,4 & 0,73 & 0,13 & 2,8 & 0,32 & 0,15 & 0,29 & 1,0 & 6,0 & 1,0 & 49 & $9 / 1$ \\
\hline
\end{tabular}


uma altura de $0,05 \mathrm{~m}$ e pesados, para determinação da produtividade (extrapolada para toneladas de colmos ha-1). Posteriormente, foram separados cinco colmos por parcela, para avaliação da qualidade industrial: teores de fibras, sólidos solúveis (Brix), Pol do caldo, Pol da cana, pureza da cana, açúcares redutores (Ar cana) e açúcar total recuperável (ATR) (Consecana 2006).

Os resultados para os atributos avaliados foram submetidos à análise de variância, pelo teste $\mathrm{F}$, e, a partir das médias obtidas, utilizou-se o teste Tukey, para o fator gesso, e foi realizada análise de regressão polinomial, para as doses de vinhaça, com significância a $10 \%$. Todos os cálculos foram efetuados utilizando-se o programa Sisvar $^{\circledR}$ (Ferreira 1999).

\section{RESULTADOS E DISCUSSÃO}

Deve-se salientar que não houve efeito significativo de interações entre gessagem e doses de vinhaça. Assim, analisando-se os resultados para os atributos químicos do solo (Tabela 3), verificou-se que a gessagem alterou significativamente os atributos químicos, na camada de $0-0,20 \mathrm{~m}$, no período de 180 dias, para $\mathrm{pH}, \mathrm{K}^{+}, \mathrm{Ca}^{2+}, \mathrm{Al}^{3+}$ e valores de saturação por bases (V\%) e por alumínio (m\%). Entretanto, este efeito não perdurou um ano após a aplicação do gesso, nesta camada, indicando que o insumo não apresentou efeito residual, neste período.
$\mathrm{O}$ aumento do valor do $\mathrm{pH}$ (Tabela 3) diferiu do que foi observado por Ernani et al. (2001), que avaliaram as alterações na fase sólida e na solução percolada em dois solos (Latossolo Bruno álico e Cambissolo Húmico álico) e dois modos de aplicação dos corretivos de acidez $\left(\mathrm{CaCO}_{3}\right.$ e $\left.\mathrm{MgCO}_{3}\right)$ e gesso agrícola, incorporado e superficial, e concluíram que, com a aplicação do gesso, o pH diminuiu, resultado, este, justificado pela hidrólise do $\mathrm{Al}^{3+}$, deslocando as cargas negativas pelo $\mathrm{Ca}^{2+}$ aplicado. Porém, segundo Raij (2008b), este efeito da gessagem é justificado pelas trocas de cátions que ocorrem na solução do solo, nas quais pode ocorrer adsorção do sulfato nas superfícies dos óxidos, favorecendo a formação de uma ponte de metal entre os dois átomos, que libera uma hidroxila $\left(\mathrm{OH}^{-}\right)$, com o $\mathrm{Ca}^{2+}$ ocupando o lugar do $\mathrm{OH}^{-}$, contrabalançando as cargas negativas do solo.

$\mathrm{O}$ potássio $\left(\mathrm{K}^{+}\right)$, em função da aplicação do gesso $\left(\mathrm{CaSO}_{4} \cdot 2 \mathrm{H}_{2} \mathrm{O}\right)$, apresentou teores reduzidos, aos 180 DAAT, em relação ao tratamento sem a aplicação de gesso (Tabela 3), corroborando o observado por Rocha (2007), que constatou que os teores de $\mathrm{K}^{+}$foram reduzidos com a aplicação do gesso e que, também, devido ao não aumento dos teores deste elemento em profundidade, pode ter ocorrido lixiviação do nutriente, pela formação de pares iônicos de cargas neutras $\left(\mathrm{K}_{2} \mathrm{SO}_{4}^{\circ}\right)$.

Quanto ao teor de $\mathrm{Ca}^{2+}$ (Tabela 3), houve incremento significativo de $5,3 \mathrm{mmol}_{\mathrm{c}} \mathrm{dm}^{-3}$, em re-

Tabela 3. Média dos atributos químicos, na camada de 0,0-0,20 m, de um Latossolo Vermelho-Amarelo cultivado com cana de segundo ciclo, aos 180 e 360 dias após a aplicação de gesso e vinhaça (Suzanápolis, SP, 2010).

\begin{tabular}{|c|c|c|c|c|c|c|c|c|c|c|c|c|c|c|}
\hline \multirow{2}{*}{\multicolumn{2}{|c|}{$\begin{array}{l}\text { Fontes de } \\
\text { variação }\end{array}$}} & \multirow{2}{*}{$\begin{array}{l}\text { P-resina } \\
\mathrm{mg} \mathrm{dm}^{-3} \\
\end{array}$} & \multirow{2}{*}{$\begin{array}{l}\text { M.O. } \\
\mathrm{g} \mathrm{dm}^{-3}\end{array}$} & \multirow{3}{*}{$\begin{array}{c}\mathrm{pH} \\
\mathrm{CaCl}_{2} \\
\end{array}$} & $\mathrm{~K}$ & $\mathrm{Ca}$ & $\mathrm{Mg}$ & $\mathrm{H}+\mathrm{Al}$ & $\mathrm{Al}^{-3}$ & \multirow{3}{*}{$\frac{\mathrm{S}_{-} \mathrm{SO}_{4}^{-2}}{\mathrm{mg} \mathrm{dm}{ }^{-3}}$} & \multirow{3}{*}{\multicolumn{2}{|c|}{$\begin{array}{cc}\mathrm{SB} & \mathrm{CTC} \\
-\mathrm{mmol}_{\mathrm{c}} \mathrm{dm}^{-3}-\end{array}$}} & \multirow{3}{*}{$\begin{array}{l}\mathrm{V} \\
\mathrm{L}\end{array}$} & \multirow{2}{*}{$\mathrm{m}$} \\
\hline & & & & & \multicolumn{5}{|c|}{$\longrightarrow \mathrm{mmol}_{\mathrm{c}} \mathrm{dm}^{-3}$} & & & & & \\
\hline \multicolumn{10}{|c|}{180 dias após os tratamentos } & & & & & \\
\hline \multirow{2}{*}{ Gesso } & sem & 5,8 & 9,9 & $4,5 \mathrm{~b}$ & $0,7 \mathrm{a}$ & $7,2 \mathrm{~b}$ & 4,7 & 22,3 & $4,2 \mathrm{a}$ & 2,3 & 12,6 & 34,9 & $36,1 \mathrm{~b}$ & $29,1 \mathrm{a}$ \\
\hline & com & 5,9 & 9,8 & $5,1 \mathrm{a}$ & $0,3 \mathrm{~b}$ & $12,5 \mathrm{a}$ & 7,1 & 20,6 & $2,4 \mathrm{~b}$ & 2,8 & 20,0 & 40,6 & $49,2 \mathrm{a}$ & $14,1 \mathrm{~b}$ \\
\hline \multicolumn{2}{|c|}{ Teste F } & $0,108^{\mathrm{ns}}$ & $0,231^{\mathrm{ns}}$ & $3,854 *$ & $3,081^{*}$ & $3,07 *$ & $2,571^{\mathrm{ns}}$ & $0,547^{\text {ns }}$ & $3,48 *$ & $0,789^{\text {ns }}$ & $0,88^{\text {ns }}$ & $1,674^{\mathrm{ns}}$ & $3,357^{*}$ & $3,159^{*}$ \\
\hline \multirow{3}{*}{ Vinhaça } & 0 & 5,6 & 9,6 & 4,9 & $0,3^{(1)}$ & 8,0 & 4,8 & 20,5 & 3,9 & 2,3 & 13,1 & 33,6 & 39,0 & $27,1^{(2)}$ \\
\hline & 100 & 5,9 & 9,7 & 4,9 & 0,6 & 9,6 & 5,9 & 21,9 & 3,8 & 3,0 & 16,1 & 38,0 & 42,3 & 22,5 \\
\hline & 200 & 6,1 & 10,1 & 4,9 & 0,6 & 11,9 & 7,0 & 22,0 & 2,3 & 2,3 & 19,5 & 41,5 & 47,0 & 15,1 \\
\hline \multicolumn{2}{|c|}{ Teste F } & $1,295^{\mathrm{ns}}$ & $0,750^{\mathrm{ns}}$ & $0,361^{\mathrm{ns}}$ & $1,95^{\mathrm{ns}}$ & $0,544^{\mathrm{ns}}$ & $0,743^{\mathrm{ns}}$ & $0,165^{\mathrm{ns}}$ & $0,668^{\mathrm{ns}}$ & $0,789^{\mathrm{ns}}$ & $0,206^{\text {ns }}$ & $1,056^{\mathrm{ns}}$ & $0,317^{\mathrm{ns}}$ & $0,686^{\mathrm{ns}}$ \\
\hline \multicolumn{2}{|c|}{ C.V. $(\%)$} & 10,6 & 8,6 & 11,8 & 9,2 & 33,3 & 28,4 & 27,0 & 44,8 & 20,1 & 40,1 & 28,2 & 21,8 & 58,5 \\
\hline \multicolumn{15}{|c|}{360 dias após os tratamentos } \\
\hline \multirow{2}{*}{ Gesso } & sem & 3,7 & 11,3 & 4,7 & 0,6 & 11,3 & 6,7 & 21,2 & 2,9 & 2,5 & 18,6 & 39,8 & 46,7 & 17,7 \\
\hline & com & 3,9 & 11,2 & 4,5 & 1,3 & 12,5 & 6,0 & 22,9 & 4,7 & 3,3 & 19,8 & 42,7 & 46,3 & 30,1 \\
\hline \multicolumn{2}{|c|}{ Teste F } & $1,667^{\mathrm{ns}}$ & $0,047^{\mathrm{ns}}$ & $0,742^{\mathrm{ns}}$ & $1,109^{\text {ns }}$ & $0,049^{\text {ns }}$ & $0,084^{\mathrm{ns}}$ & $0,559^{\mathrm{ns}}$ & $2,467^{\mathrm{ns}}$ & $5,357^{\mathrm{ns}}$ & $0,001^{\text {ns }}$ & $0,114^{\mathrm{ns}}$ & $1,007^{\mathrm{ns}}$ & $2,906^{\mathrm{ns}}$ \\
\hline \multirow{3}{*}{ Vinhaça } & 0 & $4,0^{(3)}$ & 11,5 & 4,4 & 1,3 & 9,6 & 5,0 & 21,4 & 4,4 & 15,2 & 15,9 & 37,3 & 42,6 & 30,1 \\
\hline & 100 & 3,9 & 11,2 & 5,0 & 0,9 & 18,1 & 9,9 & 21,6 & 3,0 & 28,5 & 28,9 & 50,5 & 57,2 & 16,6 \\
\hline & 200 & 3,6 & 11,1 & 4,4 & 0,7 & 8,0 & 4,2 & 23,1 & 4,1 & 13,0 & 13,0 & 36,0 & 35,8 & 24,9 \\
\hline \multicolumn{2}{|c|}{ Teste F } & $2,917 *$ & $0,329^{\mathrm{ns}}$ & $2,036^{\mathrm{ns}}$ & $0,387^{\mathrm{ns}}$ & $1,428^{\mathrm{ns}}$ & $1,867^{\mathrm{ns}}$ & $0,218^{\mathrm{ns}}$ & $0,218^{\mathrm{ns}}$ & $1,55^{\mathrm{ns}}$ & $1,55^{\mathrm{ns}}$ & $1,843^{\mathrm{ns}}$ & $1,456^{\mathrm{ns}}$ & $1,164^{\mathrm{ns}}$ \\
\hline \multicolumn{2}{|c|}{ C.V. $(\%)$} & 8,2 & 8,3 & 14,8 & 34,6 & 38,1 & 37,4 & 26,0 & 36,8 & 37,2 & 37,2 & 17,1 & 19,7 & 45,9 \\
\hline
\end{tabular}


lação à não aplicação de gesso, efeito esperado pela liberação do cátion proveniente da dissociação do $\mathrm{CaSO}_{4} \cdot 2 \mathrm{H}_{2} \mathrm{O}$, na solução do solo. Este aumento no teor de $\mathrm{Ca}^{2+}$ determinou a elevação da saturação por bases (V) para 47\%. Resultados semelhantes foram verificados por Morelli et al. (1987), que avaliaram o efeito do calcário e/ou gesso nos atributos químicos do solo e na produtividade da cana-de-açúcar, em solos arenosos álicos, e verificaram que o gesso proporcionou aumento no teor de $\mathrm{Ca}^{2+}$ em todo o perfil avaliado e promoveu a lixiviação de bases, contribuindo com a fertilidade do solo, tanto nos teores de bases trocáveis quanto na saturação por bases (V\%).

A gessagem também contribuiu para redução de $1,8 \mathrm{mmol}_{\mathrm{c}} \mathrm{dm}^{-3}$ no teor de $\mathrm{Al}^{3+}$ e, consequentemente, para a redução de $14 \%$ da saturação por alumínio ( $\mathrm{m} \%$ ) (Tabela 3), fato, este, considerado comum, pela ação do gesso na correção do elemento tóxico no solo. Esta redução dos teores de $\mathrm{Al}^{3+}$, em solos com textura arenosa e baixos valores da CTC, é propiciada pela formação dos compostos $\mathrm{AlSO}_{4}^{+}$ou $\mathrm{Al}_{2}\left(\mathrm{SO}_{4}\right)_{3}$, que podem ser lixiviados para camadas subsuperficiais, reduzindo o teor deste elemento tóxico nas superfícies dos coloides (Dias 1992).

Constatou-se, pelo teste $\mathrm{F}$, que não houve ajuste de regressão, em função das doses de vinhaça, após 180 dias de aplicação no solo (Tabela 3). Porém, foi verificado ajuste significativo para $\mathrm{o} \mathrm{K}^{+} \mathrm{e} \mathrm{m} \%$, com ajuste de equações lineares positivo para $\mathrm{K}^{+} \mathrm{e}$ negativo para $\mathrm{m} \%$ (Tabela 3 ).
Aos 360 dias após a aplicação da vinhaça (Tabela 3), o $\mathrm{P}$ diminuiu proporcionalmente, com o aumento das doses, com ajuste linear decrescente, enquanto, para os outros atributos químicos, não houve ajuste de regressão, discordando de Glória \& Orlando Filho (1983), que enumeraram os seguintes efeitos da vinhaça no solo: melhoria da estrutura física do solo e elevação do $\mathrm{pH}$, da disponibilidade de alguns íons $\left(\mathrm{K}^{+}, \mathrm{Ca}^{2+}\right.$ e $\left.\mathrm{Mg}^{2+}\right)$, da capacidade de troca catiônica e da capacidade de retenção de água. Esta discrepância pode ser justificada pela textura arenosa do solo e pelo alto índice de precipitação pluvial ocorrida durante a condução do experimento $(1.693,0 \mathrm{~mm})$, em que os nutrientes advindos da vinhaça podem ter sido lixiviados para a camada mais profunda do solo.

Na camada de 0,20-0,40 m, após 180 dias da aplicação da vinhaça e do gesso, constatou-se efeito da gessagem em subsuperfície, com incremento de

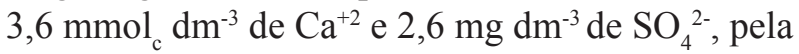
capacidade deste insumo de não receber prótons na faixa de pH agricultável do solo (Raij 2008a), contribuindo para a lixiviação do $\mathrm{Ca}^{2+} \mathrm{e} \mathrm{SO}_{4}{ }^{2-}$ para a camada subsuperficial (Tabela 4). Também foi constatada melhoria do $\mathrm{pH}$, nesta camada, aumentando 0,4 unidades, valor inferior ao recomendado para uma melhor eficiência das culturas, mas não para a cana-de-açúcar, cujo pH ideal varia em torno de 4,5 a 5,0.

A $\mathrm{m} \%$ do solo foi novamente reduzida (Tabela 4), corroborando o observado por Morelli et

Tabela 4. Média dos atributos químicos, na camada de 0,20-0,40 m, de um Latossolo Vermelho-Amarelo cultivado com cana de segundo ciclo, em função da aplicação de gesso e vinhaça, aos 180 e 360 dias (Suzanápolis, SP, 2010).

\begin{tabular}{|c|c|c|c|c|c|c|c|c|c|c|c|c|c|c|}
\hline \multirow{2}{*}{\multicolumn{2}{|c|}{$\begin{array}{c}\text { Fontes de } \\
\text { variação }\end{array}$}} & \multirow{2}{*}{$\begin{array}{l}\text { P-resina } \\
\mathrm{mg} \mathrm{dm}{ }^{-3} \\
\end{array}$} & \multirow{2}{*}{$\begin{array}{l}\text { M.O. } \\
\mathrm{g} \mathrm{dm}^{-3}\end{array}$} & \multirow{2}{*}{$\frac{\mathrm{pH}}{\mathrm{CaCl}_{2}}$} & $\mathrm{~K}$ & $\mathrm{Ca}$ & $\mathrm{Mg}$ & $\mathrm{H}+\mathrm{Al}$ & $\mathrm{Al}$ & \multirow{2}{*}{$\frac{\mathrm{S}^{-\mathrm{SO}_{4}}{ }^{-2}}{\mathrm{mg} \mathrm{dm}}$} & \multirow{2}{*}{\multicolumn{2}{|c|}{$\begin{array}{l}\mathrm{SB} \quad \mathrm{CTC} \\
-\mathrm{mmol}_{\mathrm{c}} \mathrm{dm}^{-3}-\end{array}$}} & $\mathrm{V}$ & $\mathrm{m}$ \\
\hline & & & & & - & \multicolumn{4}{|c|}{$\mathrm{mmol}_{\mathrm{c}} \mathrm{dm}^{-3}$} & & & & \multicolumn{2}{|c|}{$\%$} \\
\hline \multicolumn{15}{|c|}{180 dias após os tratamentos } \\
\hline \multirow{2}{*}{ Gesso } & sem & 5,8 & 10,1 & $4,3 \mathrm{~b}$ & 0,5 & $5,2 \mathrm{~b}$ & 3,8 & 25,5 & 6,5 & $2,8 \mathrm{~b}$ & 9,5 & 35,0 & 26,8 & $42,5 \mathrm{a}$ \\
\hline & com & 5,9 & 9,5 & $4,7 \mathrm{a}$ & 0,4 & $8,8 \mathrm{a}$ & 5,6 & 22,3 & 4,5 & $5,4 \mathrm{a}$ & 14,7 & 37,0 & 38,3 & $26,7 \mathrm{~b}$ \\
\hline \multicolumn{2}{|c|}{ Teste $\mathrm{F}$} & $0,349^{\text {ns }}$ & $4,365^{*}$ & $4,029^{*}$ & $1,34^{\mathrm{ns}}$ & $3,103 *$ & $1,673^{\mathrm{ns}}$ & $2,213^{\text {ns }}$ & $1,24^{\mathrm{ns}}$ & $4,476^{*}$ & $2,491^{\mathrm{ns}}$ & $0,955^{\mathrm{ns}}$ & $2,88^{\text {ns }}$ & $3,6^{*}$ \\
\hline \multirow{3}{*}{ Vinhaça } & 0 & 5,8 & 9,6 & 4,3 & $0,3^{(1)}$ & 6,0 & 3,6 & 25,2 & 6,6 & 4,8 & 9,9 & 35,2 & 27,5 & 41,9 \\
\hline & 100 & 5,9 & 9,8 & 4,6 & 0,5 & 9,0 & 5,6 & 22,0 & 4,9 & 3,5 & 15,1 & 37,1 & 38,1 & 29,0 \\
\hline & 200 & 6,0 & 10,0 & 4,6 & 0,5 & 6,1 & 4,6 & 24,4 & 5,0 & 4,0 & 11,2 & 35,6 & 31,8 & 32,9 \\
\hline \multicolumn{2}{|c|}{ Teste F } & $1,047^{\mathrm{ns}}$ & $1,452^{\mathrm{ns}}$ & $1,064^{\mathrm{ns}}$ & $6,5^{*}$ & $0,928^{\mathrm{ns}}$ & $0,728^{\text {ns }}$ & $0,79^{\text {ns }}$ & $0,394^{\mathrm{ns}}$ & $0,332^{\text {ns }}$ & $0,88^{\text {ns }}$ & $0,333^{\mathrm{ns}}$ & $0,852^{\text {ns }}$ & $0,465^{\text {ns }}$ \\
\hline \multicolumn{2}{|c|}{ C.V. $(\%)$} & 5,9 & 6,0 & 9,2 & 28,2 & 30,6 & 32,3 & 22,4 & 42,0 & 29,4 & 30,8 & 14,1 & 26,2 & 49,6 \\
\hline \multicolumn{15}{|c|}{360 dias após os tratamentos } \\
\hline \multirow{2}{*}{ Gesso } & sem & 3,6 & 9,7 & 4,1 & 0,4 & 4,8 & 3,0 & 28,2 & 8,8 & 3,4 & 8,2 & 35,8 & 22,8 & 50,1 \\
\hline & com & 3,6 & 9,4 & 4,2 & 0,6 & 5,4 & 3,3 & 27,7 & 9,2 & 4,7 & 9,2 & 36,8 & 25,0 & 53,6 \\
\hline \multicolumn{2}{|c|}{ Teste F } & $0,001^{\mathrm{ns}}$ & $0,167^{\mathrm{ns}}$ & $0,11^{\mathrm{ns}}$ & $0,705^{\text {ns }}$ & $0,135^{\mathrm{ns}}$ & $0,082^{\mathrm{ns}}$ & $0,029^{\mathrm{ns}}$ & $0,053^{\mathrm{ns}}$ & $1,445^{\mathrm{ns}}$ & $0,136^{\mathrm{ns}}$ & $0,403^{\mathrm{ns}}$ & $0,109^{\text {ns }}$ & $0,11^{\text {ns }}$ \\
\hline \multirow{3}{*}{ Vinhaça } & 0 & 3,6 & 9,4 & 4,0 & 0,6 & 4,0 & 2,7 & 27,8 & 9,6 & 3,4 & 7,3 & 34,8 & 20,4 & 54,7 \\
\hline & 100 & 3,8 & 10,2 & 4,3 & 0,4 & 6,9 & 3,9 & 27,9 & 7,8 & 3,5 & 11,2 & 38,2 & 29,5 & 44,9 \\
\hline & 200 & 3,4 & 9,0 & 4,1 & 0,4 & 4,5 & 2,9 & 28,1 & 9,5 & 5,2 & 7,7 & 35,9 & 21,7 & 55,9 \\
\hline \multicolumn{2}{|c|}{ Teste F } & $2,386^{\mathrm{ns}}$ & $1,468^{\mathrm{ns}}$ & $0,733^{\mathrm{ns}}$ & $0,566^{\mathrm{ns}}$ & $1,249^{\mathrm{ns}}$ & $0,376^{\text {ns }}$ & $0,006^{\mathrm{ns}}$ & $0,450^{\mathrm{ns}}$ & $1,355^{\mathrm{ns}}$ & $0,906^{\mathrm{ns}}$ & $1,8^{\mathrm{ns}}$ & $0,695^{\mathrm{ns}}$ & $0,438^{\text {ns }}$ \\
\hline \multicolumn{2}{|c|}{ C.V. $(\%)$} & 9,76 & 15,7 & 10,4 & 23,0 & 31,2 & 35,1 & 25,6 & 28,8 & 25,6 & 32,6 & 10,2 & 31,8 & 32,1 \\
\hline
\end{tabular}

* Médias seguidas de letras distintas diferem significativamente pelo teste " $\mathrm{t}$ ”, a $10 \%$; *, ** e ns: significativo a $10 \%$ e $5 \%$ e não significativo, respectivamente; (1) $\mathrm{K}=0,36+$ $0,0008 x\left(r^{2}=0,54 * *\right)$. 
al. (1987), que constataram decréscimo nos valores de $\mathrm{m} \%$, na camada de $0,25-0,50 \mathrm{~m}$, nos tratamentos em que foram aplicadas doses isoladas de gesso, avaliados após 6 meses da instalação do experimento.

Devido à precipitação pluvial muito alta (aproximadamente 1.100,0 mm, entre novembro de 2009 e janeiro de 2010; Figura 1), após a aplicação dos tratamentos (18/10/2009) em solo com textura arenosa, na camada de 0,20-0,40 m, após 180 dias, não foram obtidas respostas à gessagem e tampouco ajustes de regressão, pela aplicação da vinhaça, exceto para $\mathrm{K}^{+}$, que apresentou ajuste linear positivo, pois este é o elemento químico presente em maior concentração na vinhaça (Tabela 4).

$\mathrm{Na}$ camada de 0,40-0,60 m, a gessagem foi eficiente no fornecimento de $\mathrm{SO}_{4}^{2-}$ (Tabela 5), com aumento de $3,2 \mathrm{mmol}_{\mathrm{c}} \mathrm{dm}^{-3}$ e $6,5 \mathrm{mmol}_{\mathrm{c}} \mathrm{dm}^{-3}$, respectivamente, nos períodos de 180 e 360 dias, corroborando o observado por Morelli et al. (1998), que obtiveram incrementos de $5,9 \mathrm{mmol}_{\mathrm{c}} \mathrm{dm}^{-3} \mathrm{e}$ $2,6 \mathrm{mmol}_{\mathrm{c}} \mathrm{dm}^{-3}$, na camada de 0,50-0,75 m, aos $18 \mathrm{e}$ 27 meses após a aplicação do gesso, respectivamente, em Latossolo Vermelho-Escuro álico.

Em razão da aplicação de vinhaça na camada de 0,40-0,60 m, no período de 180 dias, não foram ajustadas regressões significativas. O mesmo resultado ocorreu aos 360 dias, antes do corte da cana soca $\left(2^{\circ}\right.$ ciclo) (Tabela 5). Apenas o $\mathrm{SO}_{4}{ }^{2-}$ apresentou efeito residual e disponibilizou $6,5 \mathrm{mmol}_{\mathrm{c}} \mathrm{dm}^{-3} \mathrm{em}$ subsuperfície, e, novamente, a aplicação de vinhaça pouco alterou a fertilidade do solo, assemelhando-se aos resultados observados na camada de $0,20-0,40 \mathrm{~m}$, devido à textura arenosa e à alta precipitação pluvial após a aplicação dos tratamentos (18/10/2009). Uma precipitação acumulada de $1.100,0 \mathrm{~mm}$, de novembro de 2009 a novembro de 2010 (Figura 1), pode ter resultado em alta lixiviação de bases e ligantes advindos tanto da vinhaça quanto do gesso.

Comparando-se a análise inicial do solo (Tabela 1) com os atributos químicos, após um ano (Tabela 3), pôde-se verificar incremento nos teores de $\mathrm{K}^{+}$e $\mathrm{Ca}^{+2}$, com aumento concomitante da $\mathrm{V}(\%)$, na camada de 0-0,20 m. Este efeito de K pode ser atribuído, em parte, à disponibilização pela decomposição da palha remanescente, ou pela ação das doses de vinhaça. Contudo, para o efeito de incremento nos teores de $\mathrm{Ca}$, além de significante redução da $\mathrm{m} \%$, por efeito da gessagem, houve melhoria nos atributos químicos do solo, como é comumente verificado em trabalhos com cana-de-açúcar, tanto em latossolo (Morelli et al. 1998) quanto em solos arenosos álicos (Morelli et al. 1987), semelhantes ao solo do presente trabalho.

Na camada de 0,40-0,60 m, no geral, observou-se redução na disponibilidade de nutrientes no solo,

Tabela 5. Média dos atributos químicos, na camada de 0,40-0,60 m, de um Latossolo Vermelho-Amarelo cultivado com cana de segundo ciclo, em função da aplicação de gesso e vinhaça, aos 180 e 360 dias (Suzanápolis, SP, 2010).

\begin{tabular}{|c|c|c|c|c|c|c|c|c|c|c|c|c|c|c|}
\hline \multirow{2}{*}{\multicolumn{2}{|c|}{$\begin{array}{l}\text { Fontes de } \\
\text { variação }\end{array}$}} & P-resina & M.O. & $\mathrm{pH}$ & $\mathrm{K}$ & $\mathrm{Ca}$ & $\mathrm{Mg}$ & $\mathrm{H}+\mathrm{Al}$ & $\mathrm{Al}^{-3}$ & \multirow{3}{*}{$\frac{\mathrm{S}_{-S O}{ }_{4}^{-2}}{\mathrm{mg} \mathrm{dm}}$} & \multirow{2}{*}{\multicolumn{2}{|c|}{$\begin{array}{cc}\mathrm{SB} & \mathrm{CTC} \\
-\mathrm{mmol}_{\mathrm{c}} \mathrm{dm}^{-3}-\end{array}$}} & \multirow{3}{*}{$\frac{\mathrm{V}}{\mathrm{L}} \mathrm{P}$} & \multirow{2}{*}{$\mathrm{m}$} \\
\hline & & $\mathrm{mg} \mathrm{dm}{ }^{-3}$ & $\mathrm{~g} \mathrm{dm}^{-3}$ & $\mathrm{CaCl}_{2}$ & \multicolumn{5}{|c|}{$\mathrm{mmol}_{\mathrm{c}} \mathrm{dm}^{-3}$} & & & & & \\
\hline \multicolumn{13}{|c|}{180 dias após os tratamentos } & & \\
\hline \multirow[t]{2}{*}{ Gesso } & sem & 5,8 & 9,7 & 4,2 & 0,4 & 4,7 & 3,1 & 28,8 & 8,5 & $3,2 \mathrm{~b}$ & 8,2 & 37,0 & 22,1 & 54,4 \\
\hline & com & 5,4 & 9,3 & 4,4 & 0,4 & 5,8 & 3,8 & 26,3 & 6,9 & $6,4 \mathrm{a}$ & 10,0 & 36,3 & 26,7 & 43,6 \\
\hline \multicolumn{2}{|c|}{ Teste F } & $2,286^{\mathrm{ns}}$ & $0,938^{\text {ns }}$ & $1,542^{\mathrm{ns}}$ & $0,025^{\text {ns }}$ & $1,168^{\mathrm{ns}}$ & $1,048^{\mathrm{ns}}$ & $1,85^{\text {ns }}$ & $1,255^{\mathrm{ns}}$ & $4,93 *$ & $1,155^{\mathrm{ns}}$ & $0,001^{\mathrm{ns}}$ & $1,329^{\text {ns }}$ & $1,435^{\text {ns }}$ \\
\hline \multirow{3}{*}{ Vinhaça } & 0 & 5,5 & 9,0 & 4,1 & 0,3 & 2,9 & 2,5 & 30,6 & 10,4 & 4,9 & 5,7 & 36,3 & 15,7 & 64,6 \\
\hline & 100 & 5,6 & 9,8 & 4,5 & 0,5 & 4,0 & 3,6 & 24,4 & 4,9 & 3,5 & 8,1 & 37,5 & 22,2 & 52,4 \\
\hline & 200 & 5,6 & 9,8 & 4,3 & 0,4 & 3,9 & 3,3 & 27,6 & 7,9 & 6,0 & 7,7 & 35,2 & 21,8 & 50,6 \\
\hline \multicolumn{2}{|c|}{ Teste F } & $0,268^{\mathrm{ns}}$ & $2,109^{\text {ns }}$ & $2,191^{\mathrm{ns}}$ & $2,553^{\mathrm{ns}}$ & $2,138^{\mathrm{ns}}$ & $2,886^{\mathrm{ns}}$ & $2,117^{\mathrm{ns}}$ & $2,06^{\mathrm{ns}}$ & $0,975^{\mathrm{ns}}$ & $1,912^{\mathrm{ns}}$ & $1,21^{\mathrm{ns}}$ & $2,21^{\mathrm{ns}}$ & $2,36^{\mathrm{ns}}$ \\
\hline \multicolumn{2}{|c|}{ C.V. (\%) } & 7,1 & 8,9 & 7,3 & 7,1 & 31,0 & 22,3 & 15,8 & 26,4 & 25,5 & 27,7 & 8,14 & 26,1 & 30,0 \\
\hline \multicolumn{15}{|c|}{360 dias após os tratamentos } \\
\hline \multirow{2}{*}{ Gesso } & sem & 3,0 & 7,2 & 4,1 & 0,3 & 4,1 & 2,0 & 24,9 & 9,9 & $5,2 \mathrm{~b}$ & 6,3 & 31,2 & 20,2 & 61,8 \\
\hline & com & 3,0 & 7,2 & 4,0 & 0,3 & 4,2 & 3,3 & 24,5 & 10,1 & $11,7 \mathrm{a}$ & 7,8 & 32,3 & 24,2 & 57,3 \\
\hline \multicolumn{2}{|c|}{ Teste F } & $1,114^{\mathrm{ns}}$ & $0,001^{\mathrm{ns}}$ & $1,379^{\mathrm{ns}}$ & $0,294^{\mathrm{ns}}$ & $0,005^{\mathrm{ns}}$ & $2,623^{\mathrm{ns}}$ & $0,047^{\mathrm{ns}}$ & $0,11^{\text {ns }}$ & $1,053^{*}$ & $0,662^{\text {ns }}$ & $0,686^{\mathrm{ns}}$ & $0,595^{\mathrm{ns}}$ & $0,242^{\text {ns }}$ \\
\hline \multirow{3}{*}{ Vinhaça } & 0 & 3,0 & 7,0 & 4,0 & 0,2 & 3,1 & 2,4 & 27,4 & 11,0 & 9,2 & 5,7 & 33,1 & 17,2 & 66,1 \\
\hline & 100 & 3,0 & 7,3 & 4,3 & 0,2 & 5,2 & 3,2 & 22,2 & 9,1 & 8,2 & 8,7 & 31,0 & 27,7 & 53,1 \\
\hline & 200 & 3,0 & 7,1 & 4,0 & 0,3 & 4,0 & 2,4 & 24,5 & 9,9 & 7,9 & 6,7 & 31,2 & 21,6 & 59,5 \\
\hline \multicolumn{2}{|c|}{ Teste F } & $0,544^{\mathrm{ns}}$ & $0,729^{\text {ns }}$ & $3,335^{\mathrm{ns}}$ & $1,176^{\mathrm{ns}}$ & $1,109^{\mathrm{ns}}$ & $0,502^{\mathrm{ns}}$ & $2,393^{\mathrm{ns}}$ & $0,476^{\mathrm{ns}}$ & $0,164^{\mathrm{ns}}$ & $1,014^{\mathrm{ns}}$ & $1,231^{\mathrm{ns}}$ & $1,323^{\mathrm{ns}}$ & $0,673^{\text {ns }}$ \\
\hline \multicolumn{2}{|c|}{ C.V. (\%) } & 13,0 & 8,8 & 6,8 & 28,2 & 26,6 & 27,1 & 19,0 & 38,7 & 28,7 & 28,7 & 9,47 & 25,8 & 24,4 \\
\hline
\end{tabular}

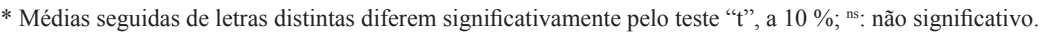


exceto para o $\mathrm{SO}_{4}^{2-}$, como já discutido anteriormente. Os teores de $\mathrm{Al}^{3+} \mathrm{e}$, concomitantemente, da $\mathrm{m} \%$, nesta camada mais profunda, não foram influenciados pelo efeito residual da gessagem, mesmo com efeito significativo no $\mathrm{SO}_{4}^{-2}$, demonstrando que poderia ter sido realizada, inicialmente, a aplicação de calcário. Além do mais, como foi empregada a adubação rotineira da Usina, esta pode não ter sido suficiente para melhorar a fertilidade química deste solo, sendo o ideal para cana soca o fornecimento de $100,0 \mathrm{~kg} \mathrm{ha}^{-1}, 30,0 \mathrm{~kg} \mathrm{ha}^{-1}$ e $130,0 \mathrm{~kg} \mathrm{ha}^{-1}$, respectivamente de $\mathrm{N}, \mathrm{P}_{2} \mathrm{O}_{5}$ e $\mathrm{K}_{2} \mathrm{O}$ (Raij \& Cantarella 1996), fato que, também, pode ser atribuído à sua baixa CTC (35,0 $\left.\mathrm{mmol}_{\mathrm{c}} \mathrm{dm}^{-3}\right)$ e teores de M.O. (11,0 $\left.\mathrm{g} \mathrm{dm}^{-3}\right)$. Assim, a prática de despalha e a não queima dos resíduos de alta relação $\mathrm{C} / \mathrm{N}$ podem contribuir com a melhoria dos atributos químicos do solo, ao longo dos demais ciclos produtivos da cana.

Os teores foliares de $\mathrm{N}, \mathrm{P}, \mathrm{K}, \mathrm{Ca}, \mathrm{Mg}$ e $\mathrm{S}$ na cana soca, aos 100 dias após a aplicação dos tratamentos (Tabela 6), não foram influenciados pela interação entre gesso e doses de vinhaça e pela aplicação exclusiva de gesso, exceto para o teor de $\mathrm{Ca}^{2+}$, que passou de 2,38 $\mathrm{g} \mathrm{kg}^{-1}$ para $2,74 \mathrm{~g} \mathrm{~kg}^{-1}$. No entanto, com relação ao teor ideal (média de 9,4-13,0 $\mathrm{g} \mathrm{kg}^{-1}$ ) para cana planta, este ainda foi muito baixo (Malavolta 2006), devido ao fato de os teores no solo já estarem baixos, conforme verificado pela análise inicial do solo, neste trabalho (Tabelas 1 e 3).

Os valores obtidos para os teores nutricionais, quando aplicadas as doses de vinhaça, apresentaram ajuste quadrático apenas para o teor de $\mathrm{Mg}^{2+}$, sendo o ponto de máxima estimado na dose de $100,0 \mathrm{~m}^{3} \mathrm{ha}^{-1}$, com aumento de $0,80 \mathrm{~g} \mathrm{~kg}^{-1}$ para $0,99 \mathrm{~g} \mathrm{~kg}^{-1}$ (Tabela 6).
Na maior dose de vinhaça, a disponibilidade deste elemento foi reduzida/inibida e não diferiu da dose zero, devido ao provável efeito de inibição competitiva entre o $\mathrm{K}^{+}$e o $\mathrm{Mg}^{2+}$ (Malavolta 2006).

Comparando-se os resultados dos teores foliares do presente trabalho (Tabela 6) aos padrões adequados, segundo Malavolta (2006), todos os teores nutricionais ficaram abaixo dos considerados adequados para a cana soca. Em relação aos teores iniciais (Tabela 1), o solo apresentou baixos teores dos nutrientes, com reduzida disponibilidade para absorção pela cultura. Além disto, a textura arenosa das camadas de 0-0,20 m e 0,20-0,40 m e a baixa CTC do solo e adubação padronizada da Usina (400,0 $\mathrm{kg} \mathrm{ha}^{-1}$ do formulado 20-0-30) podem ter contribuído para tais resultados.

A produtividade dos colmos e a qualidade tecnológica da cana colhida (Tabela 7) não demonstraram efeito dos tratamentos, diferindo dos resultados verificados nos trabalhos de Paulino et al. (2002), que estudaram, em Latossolo Vermelho argiloso, o efeito de doses de vinhaça sobre a produção agrícola e industrial em cana de $3^{\mathrm{a}}$ e $4^{\mathrm{a}}$ socas, e concluíram que a dose intermediária de $300,0 \mathrm{~m}^{3} \mathrm{ha}^{-1}$ foi a melhor, além de reduzir os custos de produção, desgaste de máquinas e gasto com combustível.

Segundo Resende et al. (2006), a aplicação de vinhaça influencia na qualidade tecnológica e produtividade da cana-de-açúcar. Como o solo apresenta textura muito arenosa, nas camadas subsuperficiais, com teores iniciais de nutrientes baixos (Tabela 1), juntamente com alta precipitação pluvial no período de desenvolvimento da cana soca, tanto a prática da gessagem como do uso de vinhaça podem ter tido

Tabela 6. Médias dos teores foliares de macronutrientes em cultivo de cana soca, em função da aplicação de gesso e vinhaça, após 100 dias (Suzanápolis, SP, 2010).

\begin{tabular}{|c|c|c|c|c|c|c|c|}
\hline \multirow{2}{*}{\multicolumn{2}{|c|}{ Fatores de Variação }} & $\mathrm{N}$ & $P$ & $\mathrm{~K}$ & $\mathrm{Ca}$ & $\mathrm{Mg}$ & $S$ \\
\hline & & \multicolumn{6}{|c|}{$\mathrm{g} \mathrm{kg}^{-1}$} \\
\hline \multirow{2}{*}{ Gesso } & sem & 8,0 & 0,45 & 9,58 & $2,38 \mathrm{~b}$ & 0,83 & 1,75 \\
\hline & com & 8,8 & 0,49 & 9,58 & $2,74 a$ & 0,88 & 1,87 \\
\hline \multicolumn{2}{|c|}{ Teste $\mathrm{F}$} & $0,128^{\mathrm{ns}}$ & $0,248^{\mathrm{ns}}$ & $0,998^{\mathrm{ns}}$ & $0,096^{*}$ & $0,433^{\mathrm{ns}}$ & $0,341^{\mathrm{ns}}$ \\
\hline \multirow{3}{*}{ Vinhaça } & 0 & 8,0 & 0,46 & 8,50 & 2,35 & $0,8^{(1)}$ & 1,6 \\
\hline & 100 & 8,9 & 0,49 & 9,94 & 2,75 & 0,98 & 1,9 \\
\hline & 200 & 8,2 & 0,46 & 10,60 & 2,59 & 0,78 & 1,9 \\
\hline Teste F & & $0,387^{\mathrm{ns}}$ & $0,796^{\mathrm{ns}}$ & $0,167^{\mathrm{ns}}$ & $0,295^{\text {ns }}$ & $0,032 *$ & $0,117^{\mathrm{ns}}$ \\
\hline C.V. $(\%)$ & & 14,8 & 18,0 & 19,8 & 19,3 & 17,7 & 17,1 \\
\hline
\end{tabular}

* Médias seguidas de letras distintas diferem significativamente pelo teste " $t$ ”, a 10\%; *, ** e ns: significativo a $10 \%$ e $5 \%$ e não significativo, respectivamente; (1) Mg = $0,8+$ $0,0038 \mathrm{x}-0,000019 \mathrm{x}^{2}(\mathrm{r}=0,99 * *)\left(\mathrm{PM}=100 \mathrm{~m}^{3} \mathrm{ha}^{-1}\right)$. 
Tabela 7. Médias dos atributos tecnológicos e de qualidade da cana soca, em função da aplicação de gesso e vinhaça (Suzanápolis, SP, 2010).

\begin{tabular}{|c|c|c|c|c|c|c|c|c|c|c|c|}
\hline \multirow{2}{*}{\multicolumn{2}{|c|}{$\begin{array}{l}\text { Fontes de } \\
\text { Variação }\end{array}$}} & $\mathrm{NCM}^{*}$ & $\mathrm{NCH}$ & $\mathrm{TCH}$ & BRIX & $\begin{array}{l}\text { Pol } \\
\text { caldo }\end{array}$ & PUR & FIB & $\begin{array}{c}\text { Pol } \\
\text { cana }\end{array}$ & AR & \multirow{2}{*}{$\begin{array}{c}\text { ATR } \\
\mathrm{kg} \mathrm{t}^{-1}\end{array}$} \\
\hline & & $\operatorname{colmos} \mathrm{m}^{-1}$ & colmos ha-1 & $\mathrm{t} \mathrm{ha}^{-1}$ & \multicolumn{6}{|c|}{ 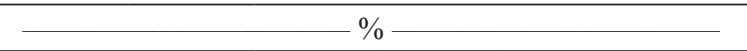 } & \\
\hline \multirow{2}{*}{ Gesso } & sem & 7,8 & 51.392 & 97,1 & 20,2 & 17,6 & 87,0 & 13,0 & 14,6 & 0,58 & 144 \\
\hline & com & 7,7 & 52.225 & 98,6 & 20,7 & 18,3 & 88,3 & 13,2 & 15,2 & 0,58 & 150 \\
\hline \multicolumn{2}{|c|}{ Teste F } & $0,034^{\mathrm{ns}}$ & $0,034^{\mathrm{ns}}$ & $0,016^{\mathrm{ns}}$ & $1,530^{\mathrm{ns}}$ & $1,36^{\mathrm{ns}}$ & $0,807^{\mathrm{ns}}$ & $0,302^{\mathrm{ns}}$ & $1,56^{\mathrm{ns}}$ & $0,077^{\mathrm{ns}}$ & $1,612^{\mathrm{ns}}$ \\
\hline \multirow{3}{*}{ Vinhaça } & 0 & 7,4 & 49.169 & 96,1 & 20,5 & 18,3 & 88,9 & 13,3 & 17,1 & 0,58 & 149 \\
\hline & 100 & 7,9 & 52.919 & 104,2 & 20,1 & 17,4 & 86,5 & 13,0 & 14,5 & 0,58 & 143 \\
\hline & 200 & 8,0 & 53.336 & 93,1 & 20,7 & 18,1 & 87,5 & 13,0 & 15,0 & 0,58 & 149 \\
\hline \multicolumn{2}{|c|}{ Teste F } & $0,173^{\mathrm{ns}}$ & $0,346^{\mathrm{ns}}$ & $0,38^{\mathrm{ns}}$ & $0,939^{\mathrm{ns}}$ & $0,922^{\mathrm{ns}}$ & $0,958^{\mathrm{ns}}$ & $0,383^{\mathrm{ns}}$ & $0,808^{\mathrm{ns}}$ & $0,30^{\text {ns }}$ & $0,774^{\mathrm{ns}}$ \\
\hline \multicolumn{2}{|c|}{ C.V. $(\%)$} & 21,3 & 21,3 & 27,0 & 4,3 & 7,3 & 3,9 & 5,3 & 7,4 & 1,3 & 7,1 \\
\hline
\end{tabular}

* Médias sem letras, nas colunas, não diferem entre si pelo teste Tukey, a $10 \%$. $\mathrm{NCM}=$ número de colmos $\mathrm{m}^{-1}$; $\mathrm{NCH}=$ número de colmos ha ${ }^{-1}$; $\mathrm{TCH}=$ produtividade de colmos; $\mathrm{Brix}=$ teor de sólidos solúveis; $\mathrm{Pol}=$ percentagem de açúcares polarizáveis; $\mathrm{PUR}=$ pureza; $\mathrm{FIB}=$ fibra; $\mathrm{AR}=$ açúcares redutores; $\mathrm{ART}=$ açúcares recuperáveis totais; $\mathrm{ATR}=$ rendimento de açúcar $\mathrm{t}^{-1}$ de cana.

seus efeitos reduzidos pela intensa lixiviação de bases (Tabelas 4 e 5), daí o não efeito dos tratamentos na produtividade de colmos e qualidade tecnológica da cana soca da variedade RB867515.

\section{CONCLUSÕES}

1. O uso do gesso e da vinhaça incrementaram os teores de $\mathrm{Ca}^{2+}, \mathrm{Mg}^{2+}$ e $\mathrm{SO}_{4}{ }^{2-}$ e os valores de $\mathrm{pH}$ e $\mathrm{V} \%$ e reduziram os teores de $\mathrm{Al}^{3+}$, na camada de 0-0,20 m e 0,20-0,40 m, e aumentaram o teor de $\mathrm{SO}_{4}^{2-}$ na camada de $0,40-0,60 \mathrm{~m}$, aos 180 e 360 dias após a aplicação dos tratamentos, respectivamente.

2. A prática da gessagem e a aplicação de vinhaça em cana soca da variedade RB 867515 de $2^{\circ}$ ciclo não resultaram em aumento na produtividade de colmos ou em sua qualidade tecnológica.

\section{REFERÊNCIAS}

BRITO, F. L.; ROLIM, M. M.; PEDROSA, E. M. R. Efeito da aplicação de vinhaça nas características químicas de solo da Zona da Mata de Pernambuco. Revista Brasileira de Ciência Agrárias, Recife, v. 4, n. 4, p. 456-462, 2009.

CANELLA, L. P. et al. Propriedades químicas de um cambissolo cultivado com cana-de-açúcar, com preservação do palhiço e adição de vinhaça por longo tempo. Revista Brasileira de Ciência do Solo, Viçosa, v. 27, n. 5, p. 935-944, 2003.

CHIARADIA, J. J. Avaliação agronômica e fluxo de gases do efeito estufa a partir de solo tratado com resíduos e cultivado com mamona (Ricinus communis L.) em área de reforma de canavial. 2005. $108 \mathrm{f}$. Tese (Doutorado em Solos e Nutrição de Plantas) - Escola Superior de Agricultura Luiz de Queiroz, Universidade de São Paulo, Piracicaba, 2005.

CONSELHO DOS PRODUTORES DE CANA-DEAÇÚCAR, AÇÚCAR E ÁLCOOL DO ESTADO DE SÃO PAULO (Consecana). Manual de instruções. 5. ed. Piracicaba: Consecana, 2006.

DIAS, L. E. Dinâmica de formas de enxofre e de cátions trocáveis em colunas de solo tratadas com diferentes doses de fósforo e gesso. 1992. $130 \mathrm{f}$. Tese (Doutorado em Engenharia Agrícola) - Universidade Federal de Viçosa, Viçosa, 1992.

EMPRESA BRASILEIRA DE PESQUISA AGROPECUÁRIA (Embrapa). Manual de métodos de análise de solo. 2. ed. Rio de Janeiro: Embrapa, 1997.

EMPRESA BRASILEIRA DE PESQUISA AGROPECUÁRIA (Embrapa). Sistema brasileiro de classificação de solos. Rio de Janeiro: Centro Nacional de Pesquisa de Solos, 2006.

ERNANI, P. R.; RIBEIRO, M. S.; BAYER, C. Modificações químicas em solos ácidos ocasionadas pelo método de aplicação de corretivos da acidez e de gesso agrícola. Scientia Agricola, Piracicaba, v. 58, n. 4, p. 825-831, 2001.

FERREIRA, D. F. Sisvar: sistemas de análise de variância para dados balanceados. In: REUNIÃO ANUAL DA REGIÃO BRASILEIRA DA SOCIEDADE INTERNACIONAL DE BIOMETRIA, 45., 2000, São Carlos. Lavras: UFLa/DEX, 1999. p. 255-258.

GLÓRIA, N. A.; ORLANDO FILHO, J. Aplicação de vinhaça como fertilizante. Boletim Técnico do Planalsucar, Araras, v. 5, n. 1, p. 5-38, 1983. 
LUCA, E. F. et al. Avaliação de atributos físicos e estoques de carbono e nitrogênio em solos com queima e sem queima de canavial. Revista Brasileira de Ciência do Solo, Viçosa, v. 32, n. 2, p. 789-800, 2008.

MALAVOLTA, E. Diagnose foliar. In: MALAVOLTA, E. Manual de nutrição mineral de plantas. São Paulo: Agronômica Ceres, 2006.

MALAVOLTA, E.; VITTI, G. C.; OLIVEIRA, S. A. Avaliação do estado nutricional das plantas: princípios e aplicações. 2. ed. Piracicaba: Associação Brasileira para Pesquisa da Potassa e do Fosfato, 1997.

MORELLI, J. L. et al. Calcário e gesso na produtividade da cana-de-açúcar e nas características químicas de um latossolo de textura média álico. Revista Brasileira de Ciência do Solo, Viçosa, v. 22, n. 2, p. 187-194, 1998.

MORELLI, J. L. et al. Efeito do gesso e do calcário nas propriedades químicas de solos arenosos álicos e na produção de cana-de-açúcar. STAB: Açúcar, Álcool e Subprodutos, Piracicaba, v. 5, n. 6, p. 24-31, 1987.

OLIVEIRA, M. W. et al. Degradação da palhada de cana-de-açúcar. Scientia Agricola, Piracicaba, v. 56, n. 4, p. 803-809, 1999.

PAULINO, A. F. et al. Produção agrícola e industrial de cana-de-açúcar submetida a doses de vinhaça. Semina: Ciências Agrárias, Londrina, v. 23, n. 2, p. 145-149, 2002.

RAIJ, B. V. Barreira química em subsolos ácidos. In: RAIJ, B. V. Gesso na agricultura. Campinas: Instituto Agronômico, 2008a. p. 12-23.

RAIJ, B. V. Gesso e atributos químicos do solo. In: RAIJ, B. V. Gesso na agricultura. Campinas: Instituto Agronômico, 2008b. p. 113-134.
RAIJ, B. V.; CANTARELLA, H. Outras culturas industriais. In: RAIJ, B. V. et al. (Orgs.). Recomendação de adubação e calagem para o Estado de São Paulo. Campinas: Instituto Agronômico, 1996. p. 233-259.

RAIJ, B. V.; QUAGGIO, J. A. Métodos de análises de solos para fins de fertilidade. Campinas: Instituto Agronômico, 1983.

RESENDE, A. S. et al. Long-term effects of pre-harvest burning and nitrogen and vinasse applications on yield of sugarcane and soil carbon nitrogen stocks on a plantation in Pernambuco, NE, Brazil. Plant and Soil, Bethlehem, v. 281, n. 1-2, p. 339-351, 2006.

ROCHA, A. T. da. Gesso mineral na melhoria do ambiente radicular da cana-de-açúcar e implicações na produtividade agricola e industrial. 2007. $69 \mathrm{f}$. Tese (Doutorado em Ciência do Solo) - Universidade Federal Rural de Pernambuco, Recife, 2007.

ROSSETO, R.; DIAS, F. L.; VITTI, C. F. Fertilidade do solo e adubação. In: DINARCO-MIRANDA, L. L.; VASCONCELOS, A. C. M.; LANDELL, M. G. de A. (Eds.). Cana-de-Açúcar. Campinas: IAC, 2008. p. 221237.

SALDANHA, E. C. M. et al. Uso do gesso mineral em latossolo cultivado com cana-de-açúcar. Revista Caatinga, Mossoró, v. 20, n. 1, p. 36-42, 2007.

UNIÃO DOS PRODUTORES DE BIOENERGIA (Udop). Características agronômicas das variedades $R B$. 2007. Disponível em: <http://www.udop.com.br/index. php?item=variedades_rb $>$. Acesso em: 20 jan. 2009. 